\title{
Clinical, Electrophysiological, and Serum Biochemical Measures of Progressive Neurological and Hepatic Dysfunction in Feline Niemann-Pick Type C Disease
}

\author{
CHARLES H. VITE, WENGE DING, CAROLINE BRYAN, PATRICIA O'DONNELL, KARYN CULLEN, DAVID ALEMAN, \\ MARK E. HASKINS, AND THOMAS VAN WINKLE \\ Departments of Clinical Studies [C.H.V., W.D., D.A., M.E.H.] and Pathobiology [C.B., P.O., K.C., M.E.H., T.W.], \\ University of Pennsylvania, School of Veterinary Medicine, Philadelphia, Pennsylvania 19104
}

\begin{abstract}
Niemann-Pick type C (NP-C) disease is a neurovisceral lysosomal storage disease characterized by neurologic dysfunction, hepatosplenomegaly, and early death. Natural history studies are very difficult to perform due to the low incidence and high heterogeneity of disease in the human population. Sixteen cats with a spontaneously occurring missense mutation in $\mathrm{NPCl}$ were evaluated over time to define the progression of neurologic and hepatic disease. Affected cats had remarkably regular onsets of specific signs of cerebellar and vestibular system dysfunction with progressive severity of dysfunction quantified by postrotatory nystagmus and brain stem auditory evoked response measures. NP-C disease cats also showed increasing serum activity of alanine aminotransferase, asparate aminotransferase, and cholesterol with advancing age. Affected cats lived to a mean age of $20.5 \pm 4.8 \mathrm{wk}$. CNS and hepatic lesions were similar to those described in human patients. These data are the first to document progressive hepatic disease in the feline model and demonstrate the importance of liver disease as part of the NP-C disease phenotype. Both neurologic and hepatic measures of disease onset and severity can be used as a baseline with which to assess the efficacy of experimental therapies of NP-C disease in the feline model. (Pediatr Res 64: 544-549, 2008)
\end{abstract}

$\mathrm{N}$ iemann-Pick type C (NP-C) disease is a neurovisceral lysosomal storage disease characterized biochemically by the endosomal/lysosomal accumulation of unesterified cholesterol, sphingomyelin, and other glycolipids, and clinically by hepatosplenomegaly, progressive neurologic dysfunction, and early death $(1,2)$. Mutations in either the $N P C 1$ or $N P C 2$ gene can result in NP-C disease with NPC1 mutations accounting for $95 \%$ of the cases $(1,3,4)$. The proteins NPC1 and NPC2 are critical for the movement of unesterified cholesterol and glycosphingolipids from the endosomal/lysosomal compartment to the Golgi apparatus, plasma membrane, and endoplasmic reticulum (5). The mechanisms by which deficient activity of these proteins results in clinical disease are not understood.

NP-C disease has an incidence of 1:150,000 and over 260 disease causing mutations have been identified (1). Natural history studies are difficult to perform due to the relatively low

Received April 15, 2008; accepted June 2, 2008.

Correspondence: Charles H. Vite, D.V.M., Ph.D., Department of Clinical Studies, School of Veterinary Medicine, University of Pennsylvania, 3900 Delancey Street, Philadelphia, PA 19104; e-mail: vite@vet.upenn.edu

Supported by Ara Parseghian Medical Research Foundation and Dana's Angels Research Trust (C.H.V.), RR02512 (M.E.H.). incidence and the high heterogeneity of disease. Onset of clinical signs ranges from the neonatal period to $59 \mathrm{y}$ of age $(1,2,6-8)$. Neurologic deterioration invariably occurs, but the onset and progression varies. Hepatosplenomegaly and neonatal jaundice are common but not always present. Several classification systems based on the age of onset of clinical signs exist with most including an early onset form (early infantile and late infantile), a juvenile-onset form, and an adult-onset form, each having a varied prevalence of specific clinical signs $(1,6,7)$. The early-onset form progresses rapidly and is often characterized by severe hepatic dysfunction and psychomotor delay during infancy with subsequent ataxia, marked spasticity, and dementia. The more slowly progressing juvenile-onset form shows intellectual impairment, hepatosplenogemegaly, supranuclear vertical gaze paresis, and ataxia, with a later onset of dementia and, variably, seizures, cataplexy, and extrapyramidal deficits. The slowly progressing late-onset form shows cognitive and psychiatric disturbances. No study recounts the progression of clinical signs in a large cohort of patients with the same mutation.

Cats with NP-C disease have a spontaneously-occurring missense mutation in NPCl (2864G-C) and clinical, neuropathological and biochemical abnormalities similar to those present in juvenile-onset patients making this model homologous to the most common form seen in human patients (9-15). The feline model has been critical for identifying the late endosomal/lysosomal accumulation of gangliosides (GM2 and GM3) and unesterified cholesterol (16), for evaluating the association of GM2 storage with meganeurite formation and abnormal dendritogenesis (13), and for correlating neuroaxonal dystrophy with neurologic dysfunction (12). The feline model has also been used to evaluate the efficacy of experimental therapy of NP-C disease $(14,17)$.

Our goal was to define and quantify the progression of CNS and hepatic disease in the feline model of NP-C disease using neurologic examinations, electrodiagnostic testing, serum biochemistry, and histopathology. This information will be used
Abbreviations: ALKP, alkaline phosphatase; ALT, alanine aminotransferase; AST, aspartate aminotransferase; BAER, brain stem auditory evoked response; CALB, calbindin; GM, ganglioside monosialo; HE, hematoxylin and eosin; HET, heterozygote; NP-C, Niemann-Pick type C; NPC1, Niemann-Pick disease type $\mathrm{C} 1$ gene or protein; PRN, post-rotatory nystagmus 
to emphasize the liver disease in feline NP-C disease, and, in the future, as a baseline with which to assess the efficacy of experimental therapies of NP-C disease in the feline model.

\section{METHODS}

Animals. Cats were raised in the animal colony of the School of Veterinary Medicine, University of Pennsylvania, under National Institutes of Health and USDA guidelines for the care and use of animals in research. Studies were approved by the University of Pennsylvania Institutional Animal Care and Use Committee. All cats were housed at $21^{\circ} \mathrm{C}$ with ad libitum food and water, 12-h light cycles, with 12-15 air changes per hour. Peripheral blood leukocytes from all cats were tested at $1 \mathrm{~d}$ of age for the NPC1 missense mutation using a PCR-based DNA test (15). Cats were identified at birth as 1) homozygous for the mutant allele (NP-C), 2) heterozygous for the mutant allele [heterozygote; (HET)], or, 3) lacking the mutant allele (wild type; WT). For this study, 16 NP-C disease, 20 heterozygotes, and 16 WT cats were evaluated.

Neurologic examination. Physical and neurologic examinations were performed weekly from birth until death. The onset and progression of signs of neurologic dysfunction (intention tremor, truncal ataxia, inability to walk, inability to stand) were identified and tracked. The effect of rotation on the number of beats of nystagmus [postrotatory nystagmus (PRN)] was determined at $8,12,16,20$, and $24 \mathrm{wk}$ of age. The number of beats of PRN was counted after moving the cats through five complete revolutions at a frequency of 34 rotations per minute using a metronome to time the rotation speed. This process was repeated three times in each direction (clockwise and counterclockwise) and the number of beats of PRN was then averaged from each time point.

Serum biochemical testing. Phlebotomy was performed at 8, 16, and 24 wk of age and serum metabolic analyses were performed using a dry chemistry analyzer (Vitros 350, Johnson and Johnson, New Brunswick, NJ).

Brain stem auditory evoked response testing (BAER). All studies were performed on cats first sedated with i.v. ketamine $(2.2 \mathrm{mg} / \mathrm{kg}$; Ketaset, Fort Dodge, IA), acepromazine maleate $(0.1 \mathrm{mg} / \mathrm{kg}$; acepromazine; BoehringerIngelheim, St. Joseph, MO), and atropine sulfate $(0.02 \mathrm{mg} / \mathrm{kg}$; Butler Animal Health Supply, Dublin, OH). Then they were anesthetized with i.v. propofol (up to $6 \mathrm{mg} / \mathrm{kg}$; Abbott Laboratories, Chicago, IL), endotracheally intubated, and maintained under anesthesia with isoflurane (IsoVet, Scherin-Plough, Omaha, NE). BAER data were recorded at 8, 16, and 24 wk of age using 12 $\mathrm{mm}, 29$ gauge subdermal needle recording electrodes and a Nicolet Viking Quest (Nicolet Biomedical, Madison, WI). The active electrode was placed over the osseous bulla of the stimulated ear, the reference electrode over the vertex of the skull, and the ground electrode over the contralateral osseous bulla. Alternating rarefaction and condensation clicks $(0.1 \mathrm{~ms}$ duration $)$ were delivered to the stimulated ear at $11.1 \mathrm{~Hz}$ using a $25-\mathrm{cm}$ plastic tube connected to a plastic earpiece. The earpiece was placed within the external ear canal. The filter settings for the amplifier were $20 \mathrm{~Hz}$ and $3 \mathrm{kHz}$. One thousand evoked responses were averaged for each tracing obtained. An amplifier sensitivity of $1 \mathrm{uV} / \mathrm{cm}$ was used to record the responses; the analysis time was $10 \mathrm{~ms}$. Central conduction time was defined as the time between the first and the fifth peak. Wave V/I amplitude was determined by dividing the amplitude of the fifth wave by the amplitude of the first wave and multiplying by 100; amplitude was measured from peak to peak. Both central conduction time and wave $\mathrm{V} / \mathrm{I}$ amplitude were used as measures of retrocochlear disease. Hearing threshold was defined as the sound intensity at which an evoked wave form was first visible.

Post mortem examination. Cats were killed using an overdose of barbiturates in accordance with the American Veterinary Medical Association guidelines. NP-C disease cats were euthanized when one of the following occurred: 1) need for full body bathing more than two consecutive days in a row (affected cats have difficulty grooming and eating due to tremors), 2) loss of $20 \%$ or more of their 4 mo-old body weight, 3) development of pressure sores due to recumbancy, or, 4) complete loss of appetite 2 days in a row. WT and heterozygous cats were euthanized between 20 and 29 wk of age. Immediately before sacrifice, each cat was given $0.5 \mathrm{~mL}$ of heparin $(1000$ units $/ \mathrm{mL}$ ) i.v. After sacrifice, the cats were perfused with $500 \mathrm{~mL}$ of $0.9 \%$ cold saline and samples of brain, liver, spleen, and lung were acquired and frozen. Next, $750 \mathrm{~mL}$ of cold $4 \%$ paraformaldehyde was perfused into the left ventricle of the heart. After perfusion, further samples of brain, liver, spleen, and lung were collected and dropped-fixed in paraformaldehyde for $48 \mathrm{~h}$. Fixed samples were paraffin-embedded, sectioned and stained with hematoxylin/eosin (HE). Immunohistochemistry for glial fibrillary acid protein (anticow GFAP; Dako, Denmark) and calbindin (antirat CALB; Swant, Switzerland) were also performed on paraffin-embedded brain sections.
Statistical methods. Mean and SD were calculated to describe the data and the unpaired 2-tailed $t$ test was used to compare data between NP-C disease and WT cats, and between WT and heterozygous cats. Significance values of $p<0.05(\dagger)$ and $p<0.001(*)$ are given.

\section{RESULTS}

Body weight. Birth weights of WT, heterozygous, and NP-C disease cats were not significantly different. However, NP-C disease cats weighed significantly $(p<0.05)$ less than WT cats beginning at 5-wk of age $(p<0.05)$ and continued to weigh less until death (Fig. 1). Cats heterozygous for the NP-C1 mutation were the same weight as WT cats at all ages.

Onset of neurologic dysfunction. NP-C disease cats had a progressive age of onset of specific nervous system signs all of which could be explained by cerebellar and vestibular dysfunction. Intention tremors and mild truncal ataxia were first seen at $6.4 \pm 0.8 \mathrm{wk}$ of age. Ataxia increased in severity and resulted in falling when running/playing at $11.8 \pm 1.9 \mathrm{wk}$ of age and increased in severity so that no cats could walk without falling at $15.3 \pm 2.6 \mathrm{wk}$ of age. Cats were unable to take even one step without falling at $17 \pm 2.1 \mathrm{wk}$ and were no longer able to stand without assistance at $19 \pm 3 \mathrm{wk}$ of age. WT and heterozygous cats developed no neurologic deficits.

$\boldsymbol{P R N}$. PRN in WT, heterozygous, and NP-C disease cats were not significantly different at 8 weeks of age. However, NP-C disease cats had significantly more PRN at 12 and 16 wk of age $(p<0.05)$, and at 20 and 24 wk of age $(p<0.001)$ weeks of age when compared with WT cats (Fig. 2). In WT and heterozygous cats, PRN significantly decreased between 8 and $12 \mathrm{wk}$ of age $(p<0.001)$. In contrast, no significant differences in PRN were found between NP-C disease cats of different ages.

$\boldsymbol{B} \boldsymbol{A} \boldsymbol{E} \boldsymbol{R}$. NP-C disease cats had significantly greater $(p<$ 0.05 ) central conduction time at 16 and 24 wk of age compared with WT cats (Fig. 3A). Although WT and heterozygous cats had a significant decrease in central conduction time between 8 and 16 wk of age, NP-C disease cats showed no similar decrease with advancing age.

NP-C disease cats also had significantly lower $(p<0.05)$ wave V/I amplitude ratio at 16 and $24 \mathrm{wk}$ of age compared

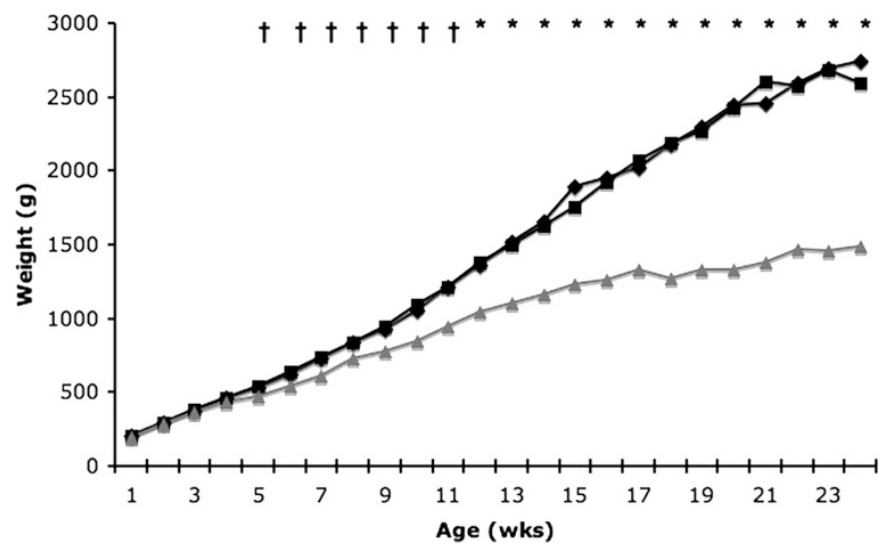

Figure 1. Weekly body weights of WT $(n=16)$, heterozygote (HET; $n=$ 20 ), and Niemann-Pick type C (NP-C; $n=16$ ) disease cats. NP-C disease cats weighed significantly less than WT cats beginning at 5-wk of age and continued to weigh less throughout life ( $\dagger p<0.05 ; * p<0.001)$. (diamond, WT; square, HET; triangle, NP-C). 


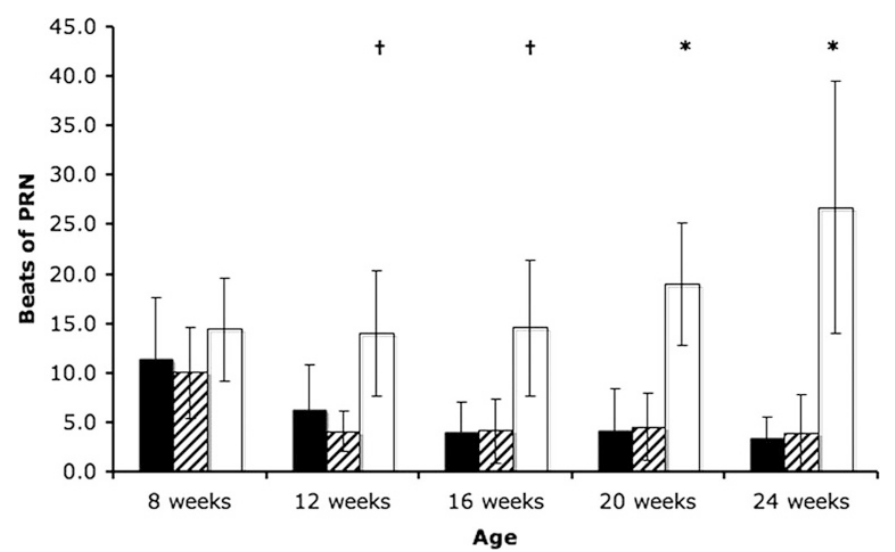

Figure 2. Postrotatory nystagmus (PRN) in WT $(n=16)$, HET $(n=20)$, and NP-C disease $(n=16)$ cats. NP-C disease cats had significantly more PRN beginning at 12 wk of age and continuing throughout life $(\dagger p<0.05$; $* p<0.001)$ compared with WT cats. A significant decrease in PRN $(p<$ 0.001 ) was seen between 8 and $12 \mathrm{wk}$ of age in WT and HET cats. (black, WT; hatched, HET; white, NP-C).

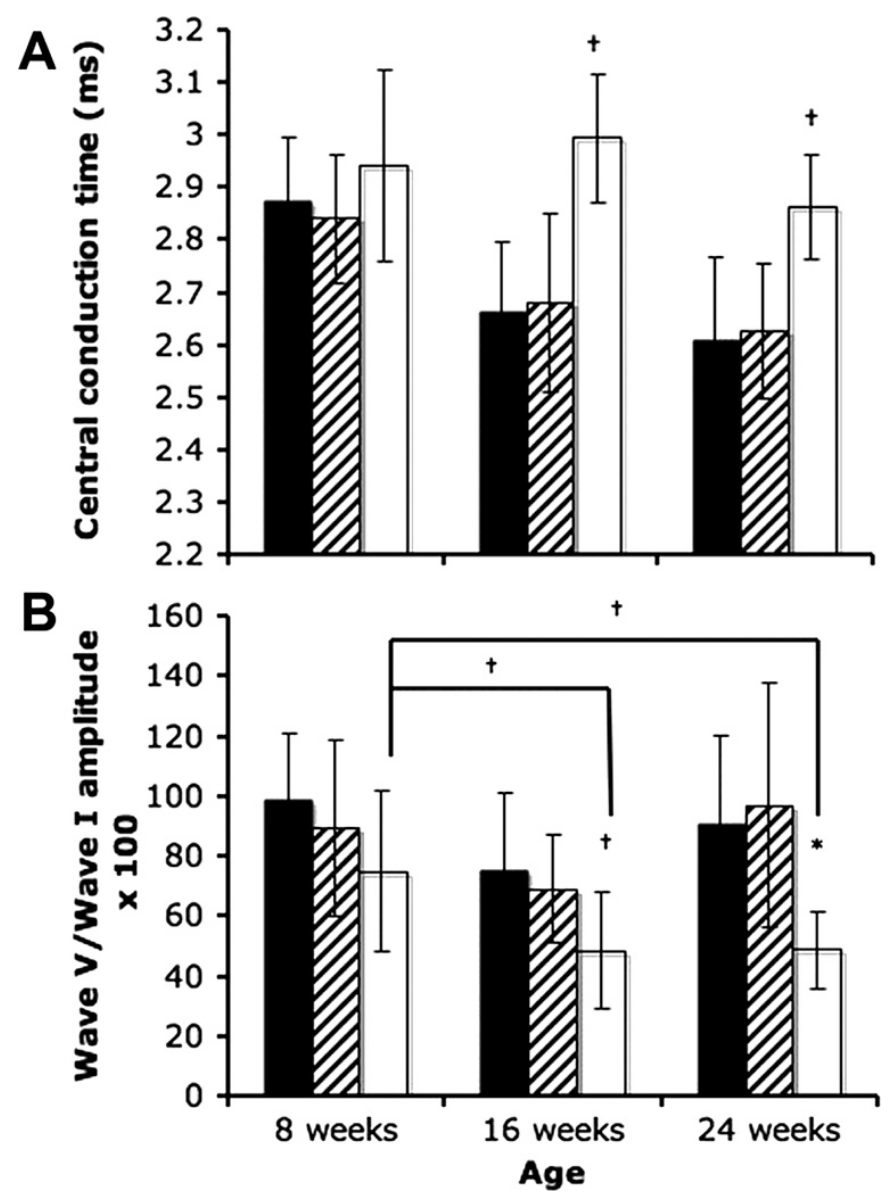

Figure 3. Central conduction time $(A)$ and wave V/I amplitude ratio $(B)$ in WT $(n=15)$, HET $(n=16)$, and NP-C disease $(n=11)$ cats. NP-C disease cats had significantly greater latency between the first and fifth wave of the BAER at 16 and 24 wk of age $(A, \dagger p<0.05)$. WT cats showed a significant decrease in central conduction time between 8 and $16 \mathrm{wk}$ and 8 and $24 \mathrm{wk}$ of age. NP-C disease cats had significantly smaller wave V/I amplitude at 16 and 24 wk of age compared with WT animals $(B, \dagger p<0.05 ; * p<0.001)$. Affected cats showed a significant decrease in wave V/I amplitude between 8 and $16 \mathrm{wk}$ of age and between 8 and $24 \mathrm{wk}$ of age. (black, WT; hatched, HET; white, NP-C). with WT cats (Fig. 3B). In NP-C disease cats, a significant decrease in V/I amplitude was found between 8 and $16 \mathrm{wk}$, and between 8 and 24 wk of age. No differences between ages were found in WT and heterozygous cats.

Finally, there was no significant difference in hearing threshold among WT, heterozygous, and NP-C disease cats at any age (data not shown).

Clinical pathology. NP-C disease cats had significantly greater $(p<0.05)$ serum concentrations of alanine aminotransferase (ALT) and aspartate aminotransferase (AST) at 8, 16, and 24 wk of age compared with WT cats (Fig. 4). As NP-C disease cats aged, significant increases in ALT and AST were found between 8 and 16 wk of age and between 8 and 24 wk of age. NP-C disease cats had significantly greater alkaline phosphatase (ALKP) activity $(p<0.05)$ at 16 and 24 wk of age compared with WT and heterozygous cats. No significant differences in these three enzymes were found between WT and heterozygous cats at any age.

At 8,16 , and 24 wk of age, NP-C disease cats had significantly lower $(p<0.05)$ serum albumin concentrations $(2.2 \pm$ $0.2 \mathrm{~g} / \mathrm{dL} ; 2.4 \pm 0.2 \mathrm{~g} / \mathrm{dL} ; 2.6 \pm 0.3 \mathrm{~g} / \mathrm{dL}$ ) compared with WT cats $(2.6 \pm 0.4 \mathrm{~g} / \mathrm{dL} ; 2.9 \pm 0.3 \mathrm{~g} / \mathrm{dL} ; 3.1 \pm 0.1 \mathrm{~g} / \mathrm{dL})$. NP-C disease cats also had significantly greater $(p<0.05)$ serum cholesterol concentrations at 8,16 , and 24 wk of age, and significantly greater serum bilirubin concentrations at 16 and 24 wk of age compared with WT cats (Table 1). Serum cholesterol concentrations in NP-C disease cats were significantly greater at 16 and 24 wk of age compared with 8 wk-old NP-C disease cats $(p<0.05)$. No significant differences were found in serum albumin, cholesterol, or total bilirubin concentrations between WT and heterozygous cats at any age.

Finally, to assess the cause of the decreased albumin and the function of the liver in NP-C disease cats, urine protein/ creatinine ratios and serum bile acids were measured. These tests were only run for two 25 wk-old NP-C disease cats. Urine protein/creatinine ratio was normal $(0.15$ and 0.18 ; reference range $<0.2)(18)$. Mild elevations in both pre-

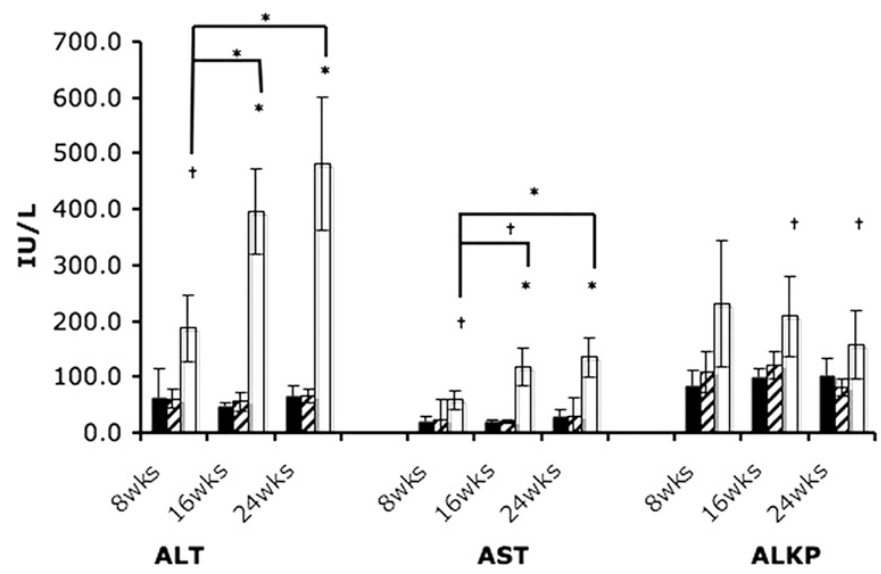

Figure 4. Hepatic enzyme activity in WT $(n=13), \operatorname{HET}(n=18)$, and NP-C disease $(n=16)$ cats. NP-C disease cats had significantly greater ALT and AST activity at all ages examined compared with WT cats $\left(\dagger p<0.05\right.$; $\left.{ }^{*} p<0.001\right)$. Significant increases in ALT and AST were found in NP-C disease cats over time between 8 and 16 wk of age and between 8 and 24 wk of age. Significantly greater ALKP activity was found in NP-C disease cats at 16 and 24 wk of age compared with WT cats. (black, WT; hatched, HET; white, NP-C). 
Table 1. Serum cholesterol and total bilirubin concentrations $(\dagger p<0.05 ; * p<0.001)$

\begin{tabular}{|c|c|c|c|}
\hline & WT & Heterozygote & NP-C disease \\
\hline \multicolumn{4}{|c|}{ Cholesterol (mg/dL) } \\
\hline 8 weeks & $146 \pm 50$ & $132 \pm 38$ & $170 \pm 35 \dagger$ \\
\hline 16 weeks & $105 \pm 10$ & $112 \pm 13$ & $244 \pm 34 *+$ \\
\hline 24 weeks & $115 \pm 19$ & $120 \pm 49$ & $237 \pm 29 *$ \\
\hline \multicolumn{4}{|c|}{ Total bilirubin $(\mathrm{mg} / \mathrm{dL})$} \\
\hline 8 weeks & $0.5 \pm 0.2$ & $0.5 \pm 0.2$ & $0.6 \pm 0.2$ \\
\hline 16 weeks & $0.4 \pm 0.1$ & $0.3 \pm 0.1$ & $0.9 \pm 0.4^{*}$ \\
\hline 24 weeks & $0.4 \pm 0.1$ & $0.3 \pm 0.1$ & $0.9 \pm 0.1^{*}$ \\
\hline
\end{tabular}

prandial $(8.6$ and $22.0 \mu \mathrm{M}$; reference $<2.0 \mu \mathrm{M})$ and postprandial bile acids (18.4 and $37.8 \mu \mathrm{M}$; reference $<10 \mu \mathrm{M})$ were found.

Survival times. Mean survival time for NP-C disease cats was $20.5 \pm 4.8 \mathrm{wk}$ (range, 11-29 wk). All cats were euthanized due to progressive tremors that made it difficult for them to eat and groom. At the time of euthanasia, no cats were able to remain sternal without being supported.

Histology. Hepatomegaly was noted at gross post mortem examination in all NP-C disease cats. The liver was weighed in two affected (121 and $132 \mathrm{~g}$; both $12 \%$ body weight) and two WT cats (63.9 and $71 \mathrm{~g} ; 3$ and $2 \%$ body weight). Similarly, the brain was weighed in two affected (20.5 and $21 \mathrm{~g}$; both $2 \%$ body weight) and two WT cats (29.2 and $30 \mathrm{~g}$; 0.9 and $1 \%$ body weight). Histologic evaluation showed that the cytoplasm of hepatocytes and Kuppfer cells had severe and extensive vacuolization and dilated hepatic sinusoids containing clusters of macrophages with granular to foamy vacuolated cytoplasm. The brain had multifocal neuronal cytoplasmic vacuolization with gliosis. Neuronal necrosis was seen with severe loss of Purkinje cells visible on both hematoxylin/eosin and calbindin-stained slides (Fig. 5). Thinning of the granular cell layer of the cerebellum was also seen. The white matter of the cerebellum showed swollen axons with dilated myelin sheaths, numerous spheroids, and myelomacrophages. Astrogliosis of both the gray and white matter was present and numerous swollen macrophages were seen surrounding vessels. The spleen and lung showed multifocal histiocytosis with macrophages containing small clear vacuoles.

\section{DISCUSSION}

Feline NP-C1 disease is due to a missense mutation, a guanine to cytosine substitution (2864G-C; C955S), which is similar to most of the juvenile onset patients (15). Previous studies performed in seven NP-C disease cats with the same mutation described signs of cerebellar dysfunction developing at approximately 8 wk of age with most animals either dying or being euthanized due to the severity of disease progression before 8 mo of age $(9-11)$. No descriptive statistical data for age of onset or progression of signs of neurologic dysfunction were previously published. Our study showed a regular onset of progressive signs of cerebellar and vestibular system dysfunction beginning at about $6 \mathrm{wk}$ of age that progressed until

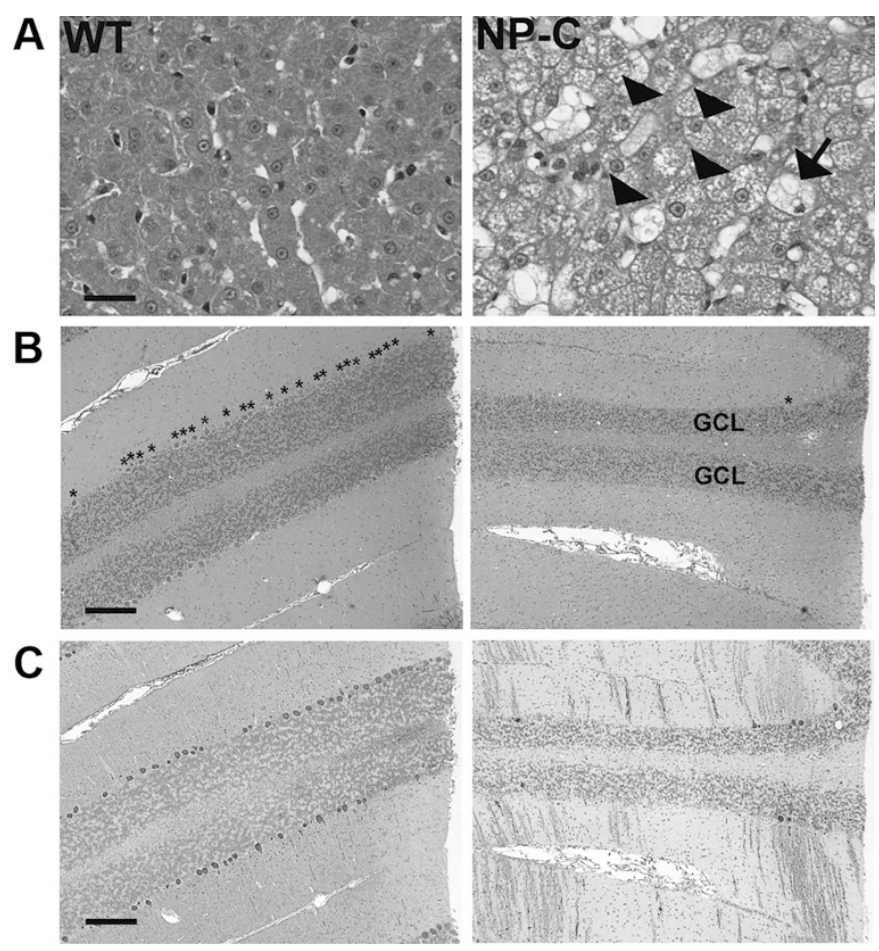

Figure 5. Hepatic $(\times 400)$ and cerebellar $(\times 50)$ histology of age-matched WT and NP-C disease cats. A, Liver from a 22 wk old NP-C disease cat showed vacuolization of both Kuppfer cells (arrow) and hepatocytes (arrowheads). A decrease in cerebellar Purkinje cell number was seen on both $(B)$ hematoxylin/eosin (*above Purkinje cells) and $(C)$ calbindin stained sections (Purkinje cell soma and process stained brown). Thinning of the granular cell layer (GCL) was also present. Bar $=25$ um (liver). $\mathrm{Bar}=200 \mathrm{um}$ (cerebellum).

cats were unable to remain in sternal recumbancy. A quantitative measure of vestibular dysfunction was developed based on the number of beats of PRN. NP-C disease cats had significantly greater PRN beginning at $12 \mathrm{wk}$ of age and this difference between affected and WT cats continued until at least $24 \mathrm{wk}$ of age. Although there was an apparent trend for increasing beats of PRN in NP-C disease cats with increasing age, the large variance found in affected cats did not make this increase statistically significant. Interestingly, WT and heterozygous cats had more PRN at 8 wk of age than at later ages, which likely represents ongoing development of the vestibular system. In contrast, NP-C disease cats showed no decrease in PRN between 8 and $12 \mathrm{wk}$ of age. Central conduction time is a measure of transit time from the earliest stimulation of the acoustic nerve to stimulation of the inferior colliculus (19). NP-C disease cats showed a delay in central conduction time at 16 and 24 wk of age compared with WT cats. Like the PRN data, WT cats showed a significant decrease in central conduction time developing after 8 wk of age that likely represents continued maturation of the acoustic system. A decrease in the wave V/I amplitude ratio has been used an indicator of retrocochlear (cranial nerve VII or brain stem) pathology (20). NP-C disease cats developed a significant decrease in this ratio between 8 and 16 wk of age. Taken together, the decrease in central conduction time and in amplitude of waves V/I suggest eighth nerve and/or brain stem disease (20), and the lack of a difference in hearing threshold in NP-C disease 
cats compared with WT cats suggests the absence of functionally significant cochlear pathology.

In addition to cerebellar and vestibular dysfunction, human patients may show vertical supranuclear gaze palsy, dysarthria, dysphagia, cataplexy, seizures, and cognitive and psychiatric disturbances (2). All cats maintained oculocephalic eye movements in all directions; however, NP-C disease cats did not readily follow targets in either the horizontal or vertical direction. It could not be determined whether the failure of "tracking" represented an abnormality of voluntary control of ocular muscles or a disinterest associated with the severity of disease. Affected cats showed no obvious abnormalities of behavior that could be interpreted as cognitive or psychiatric dysfunction, however, impairment of motor ability due to cerebellar dysfunction was severe and, therefore, limited the behavioral observations that could be made. No generalized seizures or cataleptic episodes were observed, however, as affected cats aged, they developed abnormal episodic rhythmic chewing motions when held which may indicate focal seizures. Electroencephalographic studies are in progress to evaluate further the presence or absence of epileptiform activity in affected cats.

Elevations in serum liver enzymes, bile acids, total bilirubin, and cholesterol, and decreased serum albumin have been previously reported in feline NP-C disease cats but there is are no data evaluating changes in these serum activity and concentrations over time (14). The activity of the enzymes ALT and AST were significantly greater in NP-C disease cats at all ages examined compared with WT cat. ALT is a cytosolic enzyme with a half life of hours in the cat (21). It is considered to be specific for increases in hepatocellular permeability and its rise over time in NP-C disease cats suggests ongoing and progressively worsening hepatocellular damage. AST is located in both the cytosol and mitochondria and serum levels increase with hepatocellular or muscle disease. Both increases in ALT and AST suggest progressive hepatocellular disease. Finding decreased albumin, without albuminuria, and increased bile acids in affected cats confirms the presence of hepatocellular dysfunction. In contrast, serum ALKP activity does not increase with hepatocellular permeability but increases with cholestasis and with osteoblastic activity associated with growth. The presence of concurrent hyperbilirubinemia with increased ALKP suggests cholestasis although no icterus was identified in these cats; no bone pathology in NP-C disease has been described. Although histologic evaluation of the liver showed no ductular or canalicular bile stasis, hepatocellular bile accumulation is difficult to see histologically in cats and we, therefore, suggest that affected cats have some degree of abnormal bilirubin metabolism or hepatobiliary bile stasis.

Progressive neonatal liver failure and cholestasis have been described in neonatal NP-C disease in human patients (2225). In one study of 52 children, 34 had cholestatic liver disease and approximately half of these children had persistent elevations of liver enzymes although neurologic dysfunction remained the predominant disease manifestation (24). In mice with NP-C disease, progressive increases in ALT and AST have been reported to occur throughout life (26). The presence of increasing serum liver enzyme activity and of liver dysfunction in the feline model supports that liver disease is an important part of NP-C disease in the feline model, as it is in the neonatal disease in human patients and in the murine model.

NP-C disease cats were euthanized when the severity of the ataxia impaired urination/defecation, eating, and grooming to the extent that cats required repetitive bathing. No cats lost substantial weight, developed pressure sores, or were anorexic. The majority of affected cats were continuing to gain weight at the time of death albeit much more slowly than unaffected littermates. With aggressive nursing care, it is expected that affected cats could be maintained for longer periods of time.

Finally, cats heterozygous for the $\mathrm{NPCl}$ mutation demonstrate abnormal cholesterol and sphingomyelin concentration within the brain as well as evidence of mild neuropathology (27). Our study shows that these biochemical and histologic abnormalities did not result in measurable abnormalities using neurologic examination, electrodiagnostic testing, or serum metabolite analyses.

The availability of a well-characterized feline NP-C disease model has several benefits. The feline model has a missense mutation with a juvenile onset of neurologic signs making it homologous to the most common disease form seen in human patients. The size of the cat allows for safe and repeated sampling of blood and other body fluids for biochemical and pharmacological measurements, and the large size of the cat brain permits the study of regional neuropathology using imaging as has been reported in other feline disease models (28). The lesions of the CNS including the abnormality of ectopic dendritogenesis, more closely resemble those found in human patients than do those in the murine model (16). Finally, the ability to genotype animals at birth, and the availability of ante mortem quantitative measures of disease progression will prove useful for evaluating the efficacy of experimental therapies.

Acknowledgment. We thank Dr. Mary Beth Callan for her critical advice on the manuscript.

\section{REFERENCES}

1. Vanier MT, Millat G 2003 Niemann-Pick disease type C. Clin Genet 64:269-281

2. Patterson MC, Vanier MT, Suzuki K, Morris JA, Carstea E, Neufeld EB, Blanchette Mackie EJ, Pentchev PG 2001 Niemann-Pick disease type C: a lipid trafficking disorder. In: Valle D, Beaudet AL, Vogelstein B, Kinzler KW, Antonarakis SE, Ballabio A (eds) Metabolic and Molecular Bases of Inherited Disease. 8th ed. New York: McGraw-Hill Companies. Available at: http://www.ommbid.com

3. Carstea ED, Morris JA, Coleman KG, Loftus SK, Zhang D, Cummings C, Gu J, Rosenfeld MA, Pavan WJ, Krizman DB, Nagle J, Polymeropoulos MH, Sturley SL, Ioannou YA, Higgins ME, Comly M, Cooney A, Brown A, Kaneski CR, BlanchetteMackie EJ, Dwyer NK, Neufeld EB, Chang TY, Liscum L, Strauss JF III, Ohno K, Zeigler M, Carmi R, Sokol J, Markie D, O’Neill RR, van Diggelen OP, Elleder M, Patterson MC, Brady RO, Vanier MT, Pentchev PG, Tagle DA 1997 Niemann-Pick C1 disease gene: homology to mediators of cholesterol homeostasis. Science 277:228-231

4. Naureckiene S, Sleat DE, Lackland H, Fensom A, Vanier MT, Wattiaux R, Jadot M, Lobel P 2000 Identification of HE1 as the second gene of Niemann-Pick C disease. Science 290:2298-2301

5. Li H, Repa JJ, Valasek MA, Beltroy EP, Turley SD, German DC, Dietschy JM 2005 Molecular, anatomical, and biochemical events associated with neurodegeneration in mice with Niemann-Pick type C disease. J Neuropathol Exp Neurol 64:323-333

6. Fink JK, Filling-Katz MR, Sokol J, Cogan DG, Pikus A, Sonies B, Soong B, Pentchev PG, Comly ME, Brady RO 1989 Clinical spectrum of Niemann-Pick disease type C. Neurology 39:1040-1049 
7. Iturriaga C, Pineda M, Fernandez-Valero EM, Vanier MT, Coll MJ 2006 NiemannPick $\mathrm{C}$ disease in Spain: clinical spectrum and development of a disability scale. J Neurol Sci 249:1-6

8. Higgins JJ, Patterson MC, Dambrosia JM, Pikus AT, Pentchev PG, Sato S, Brady RO, Barton NW 1992 A clinical staging classification for type C Niemann-Pick disease. Neurology 42:2286-2290

9. Lowenthal AC, Cummings JF, Wenger DA, Thrall MA, Wood PA, de Lahunta A 1990 Feline sphingolipidosis resembling Niemann-Pick disease type C. Acta Neuropathol 81:189-197

10. Brown DE, Thrall MA, Walkley SU, Wenger DA, Mitchell TW, Smith MO, Royals KL, March PA, Allison RW 1994 Feline Niemann-Pick disease type C. Am J Patho $144: 1412-1415$

11. Munana KR, Luttgen PJ, Thrall MA, Mitchell TW, Wenger DA 1994 Neurologica manifestations of Niemann-Pick disease type C in cats. J Vet Intern Med 8:117-121

12. March PA, Thrall MA, Brown DE, Mitchell TW, Lowenthal AC, Walkley SU 1997 GABAergic neuroaxonal dystrophy and other cytopathological alterations in feline Niemann-Pick disease type C. Acta Neuropathol 94:164-172

13. Zervas M, Dobrenis K, Walkley SU 2001 Neurons in Niemann-Pick disease type C accumulate gangliosides as well as unesterified cholesterol and undergo dendritic and axonal alterations. J Neuropathol Exp Neurol 60:49-64

14. Somers KL, Brown DE, Fulton R, Schultheiss PC, Hamar D, Smith MO, Allison R, Connally HE, Just C, Mitchell TW, Wenger DA, Thrall MA 2001 Effects of dietary cholesterol restriction in a feline model of Niemann-Pick type $\mathrm{C}$ disease. J Inherit Metab Dis 24:427-436

15. Somers KL, Royals MA, Carstea ED, Rafi MA, Wenger DA, Thrall MA 2003 Mutation analysis of feline Niemann-Pick C1 disease. Mol Genet Metab 79:99-103

16. Walkley SU, Suzuki K 2004 Consequences of NPC1 and NPC2 loss of function in mammalian neurons. Biochim Biophys Acta 1685:48-62

17. Zervas M, Somers KL, Thrall MA, Walkley SU 2001 Critical role for glycosphingolipids in Niemann-Pick disease type C. Curr Biol 11:1283-1287
18. King JN, Tasker S, Gunn-Moore DA, Strehlau G; BENRIC (benazepril in renal insufficiency in cats) Study Group 2007 Prognostic factors in cats with chronic kidney disease. J Vet Intern Med 21:906-916

19. Buchwald JS, Huang CL 1975 Far-field acoustic response: origins in the cat. Science 189:382-384

20. Silman S, Silverman CA 1991 Braistem auditory evoked potentials. In: Auditory Diagnosis: Principles and Applications. San Diego: Academic Press, pp 151-297

21. Lassen ED 2004 Laboratory evaluation of the liver. In: Thrall MA (ed) Veterinary Hematology and Clinical Chemistry. Philadelphia: Lippincott Williams, Wilkins, pp 355-375

22. Rutledge JC 1989 Progressive neonatal liver failure due to type C Niemann-Pick disease. Pediatr Pathol 9:779-784

23. Vanier MT, Wenger DA, Comly ME, Rousson R, Brady RO, Pentchev PG 1988 Niemann-Pick disease group $\mathrm{C}$ : clinical variability and diagnosis based on defective cholesterol esterification. A collaborative study on 70 patients. Clin Genet 33:331348

24. Kelly DA, Portmann B, Mowat AP, Sherlock S, Lake BD 1993 Niemann-Pick disease type $\mathrm{C}$ : diagnosis and outcome in children, with particular reference to liver disease. J Pediatr 123:242-247

25. Ashkenazi A, Yarom R, Gutman A, Abrahamov A, Russell A 1971 Niemann-Pick disease and giant cell transformation of the liver. Acta Paediatr Scand 60:285-294

26. Beltroy EP, Richardson JA, Horton JD, Turley SD, Dietschy JM 2005 Cholesterol accumulation and liver cell death in mice with Niemann-Pick type $\mathrm{C}$ disease. Hepatology 42:886-893

27. Brown DE, Thrall MA, Walkley SU, Wurzelmann S, Wenger DA, Allison RW, Just CA 1996 Metabolic abnormalities in feline Niemann-Pick type C heterozygotes. J Inherit Metab Dis 19:319-330

28. Vite CH, Magnitsky S, Aleman D, O'Donnell P, Cullen K, Ding W, Pickup S, Wolfe JH, Poptani H 2008 Apparent diffusion coefficient reveals gray and white matter disease, and T2 mapping detects white matter disease in the brain in feline alphamannosidosis. AJNR Am J Neuroradiol 29:308-313 